\title{
Correlations between Gene Resistant Markers and Second-Line Anti-TB Drug Resistance in Pre-XDR and XDR-TB Patients
}

\author{
Risara Jaksuwan1, Prasit Tharavichikul'2, Charoen Chuchottaworn ${ }^{3}$, Jayanton Patumanond4, \\ Piyada Kunawararak ${ }^{5}$, Jongkolnee Settakorn ${ }^{6 *}$
}

${ }^{1}$ Clinical Epidemiology Unit, Faculty of Medicine, Chiang Mai University, Chiang Mai, Thailand

${ }^{2}$ Department of Microbiology, Faculty of Medicine, Chiang Mai University, Chiang Mai, Thailand

${ }^{3}$ Division of Respiratory Medicine, Chest Disease Institute, Nonthaburi, Thailand

${ }^{4}$ Faculty of Medicine, Thammasat University, Bangkok, Thailand

${ }^{5}$ Office of Disease Prevention and Control 10, Chiang Mai, Thailand

${ }^{6}$ Department of Pathology, Faculty of Medicine, Chiang Mai University, Chiang Mai, Thailand

Email: *jsettakorn@gmail.com

How to cite this paper: Jaksuwan, R., Tharavichikul, P., Chuchottaworn, C., Patumanond, J., Kunawararak, P. and Settakorn, J. (2017) Correlations between Gene Resistant Markers and Second-Line Anti-TB Drug Resistance in Pre-XDR and XDR-TB Patients. Journal of Tuberculosis Research, $\mathbf{5}$, 178-188.

https://doi.org/10.4236/jtr.2017.53020

Received: July 2, 2017

Accepted: August 21, 2017

Published: August 24, 2017

Copyright $\odot 2017$ by authors and Scientific Research Publishing Inc. This work is licensed under the Creative Commons Attribution International License (CC BY 4.0).

http://creativecommons.org/licenses/by/4.0/

\section{c) (i) Open Access}

\begin{abstract}
Background: Extensively drug resistant tuberculosis (XDR-TB) is a serious problem in public health and XDR-TB patients usually develop from multi-drug resistance tuberculosis (MDR-TB) and pre-XDR-TB. The rapid molecular test for drug susceptibility testing (DST) can be used for early detection to prevent XDR-TB. Methods: We examined 34 clinical Mycobacterium tuberculosis ( $M$. tuberculosis) isolates from MDR/XDR-TB patients in the upper north of Thailand that were identified with drug susceptibility profiles by indirect agar proportion method from 2005-2012. Our study investigated the genetic mutations in gyrA for ofloxacin resistance and rrs for kanamycin resistance. The genetic mutations and drug susceptibility test results were analyzed using the exact test. Results: The majority of the ofloxacin resistance was detected in gyrA 21, gyrA 70, gyrA 87, gyrA 102, gyrA 162, and gyrA 187 were at $0 \%$, $12.5 \%, 37.5 \%, 0 \%, 50.0 \%$ and $25.0 \%$ sensitivity, respectively, and at 96.2 , $96.2 \%, 20.1 \%, 96.2 \%, 57.7 \%$ and $61.5 \%$ specificity, respectively. Kanamycin resistance was found in rrs 512, rrs 241, rrs 223, rrs 414 and rrs 408 at $16.7 \%$, $0 \%, 0 \%, 16.7 \%$ and $16.7 \%$ sensitivity, respectively, and at $96.4 \%, 92.9 \%, 82.1 \%$, $82.1 \%$ and $71.4 \%$ specificity, respectively. This study found no significant correlation between gyrA mutations and ofloxacin resistance and also no correlation between the rrs gene and kanamycin resistance. Conclusion: These primer sequences and PCR products in our study such as gyrA and rrs might be unsuitable to detect ofloxacin and kanamycin resistance in the upper
\end{abstract}


north of Thailand.

\section{Keywords}

XDR-TB, Pre-XDR-TB, Ofloxacin, gyrA, Kanamycin, rrs

\section{Introduction}

The emerging and increasing extensively drug resistant tuberculosis (XDR-TB) is a serious problem in public health. XDR-TB is caused by the Mycobacterium tuberculosis (M. tuberculosis), which resists up to at least four drugs such as isoniazid (INH), rifampicin (RIF), any fluoroquinolone and at least one of three second-line drug injections: capreomycin, kanamycin and amikacin [1]. The emergence of XDR-TB is caused by many factors, in conjunction with the low laboratory capacity to diagnose XDR-TB; thus, resulting in ineffective control of the spread of the disease and ineffective initial treatment [1] [2] [3]. Treatment of XDR-TB is very complicated and limited because first and second-line antiTB drugs cannot be used, leading to a long duration, expensive costs, less effective outcomes and many side effects [4] [5].

XDR-TB normally develops from multi-drug resistant tuberculosis (MDR-TB) to pre-extensively drug resistant tuberculosis (pre-XDR-TB), and then turns to XDR-TB. The definition of pre-XDR-TB is a disease caused by the M. tuberculosis strain that resists isoniazid and rifampin and either a fluoroquinolone or a second-line injectable drug, but not both [6]. Rapid diagnosis of resistance to second-line anti-TB strains is extremely important leading to effective treatment and prevention of the spread of pre-XDR/XDR strains [7]. Molecular assays, such as MTBDRsl, are rapid diagnosis tests to detect second-line drug resistance based on drug resistant gene mutations [4]. Analyzing the mutation types and frequencies among local strains are fundamentally important for evaluating the usefulness of these molecular tools.

Today, commercially available molecular tests, endorsed by the WHO, can detect only rifampicin and isoniazid, which means they can detect MDR-TB but not pre-XDR and XDR-TB [1] [8]. Related studies have shown specificities of gene mutations in predicting kanamycin [9] [10] [11] and ofloxacin resistance [11] [12] [13] [14]. Many studies have been conducted on genotypic and phenotypic characteristics of drug resistant $M$. tuberculosis, however, this kind of study is rare in Thailand [15]. Better understanding of the molecular mechanisms of drug resistance in pre-XDR-TB and XDR-TB strains can contribute to the improvement of existing techniques in detecting drug resistance and help to explore new target sites and develop new diagnostic tools.

The objective of this study was to determine the association (relationship) between mutation in gyrA and resistance to ofloxacin as well as mutation in rrs and resistance to kanamycin in MDR, pre-XDR and XDR TB patients in the 
northern region of Thailand.

\section{Materials and Methods}

\subsection{Bacterial Isolates and Drug Susceptibility Testing}

A total of $34 \mathrm{MDR}$ and XDR M. tuberculosis isolates were collected by the Office of Disease Prevention and Control Region 10 (DPC 10) from patients with MDR/XDR-TB in the upper north of Thailand from 2005 to 2012. The identification of MDR/XDR-TB isolates was performed conventionally using the indirect proportion method on agar based medium with concentrations of isoniazid $(0.2 \mu \mathrm{g} / \mathrm{ml})$, rifampicin $(40 \mu \mathrm{g} / \mathrm{ml})$, etambutol $(2 \mu \mathrm{g} / \mathrm{ml})$ and streptomycin $(4$ $\mu \mathrm{g} / \mathrm{ml})$ as shown in Figure 1 [16]. When they resisted isoniazid and rifampicin, then ofloxacin $(2.0 \mu \mathrm{g} / \mathrm{ml})$ and kanamycin $(30 \mu \mathrm{g} / \mathrm{ml})$ susceptibility was tested on middle brook medium using the proportion method. All 34 isolates were subcultured in $5 \mathrm{ml}$ of 7H9 broth with PANTA supplementation and 3\% Ogawabased medium. In all, 34 isolates were re-grown and tested for their phenotypes using first and second-line drugs (isoniazid, rifampicin, ofloxacin and kanamycin) by the proportion method with LJ medium as shown in Figure 1 [17].

Drug susceptibility testing (DST) was conducted by the indirect agar proportion method on two of LJ medium and bacilli suspension of $10^{-2}$ and $10^{-4} \mathrm{mg} / \mathrm{ml}$ for inoculation. The LJ medium were inoculated with following drugs isoniazid $(0.2 \mu \mathrm{g} / \mathrm{ml})$, rifampicin $(40.0 \mu \mathrm{g} / \mathrm{ml})$, ofloxacin $(2.0 \mu \mathrm{g} / \mathrm{ml})$, and kanamycin $(30$

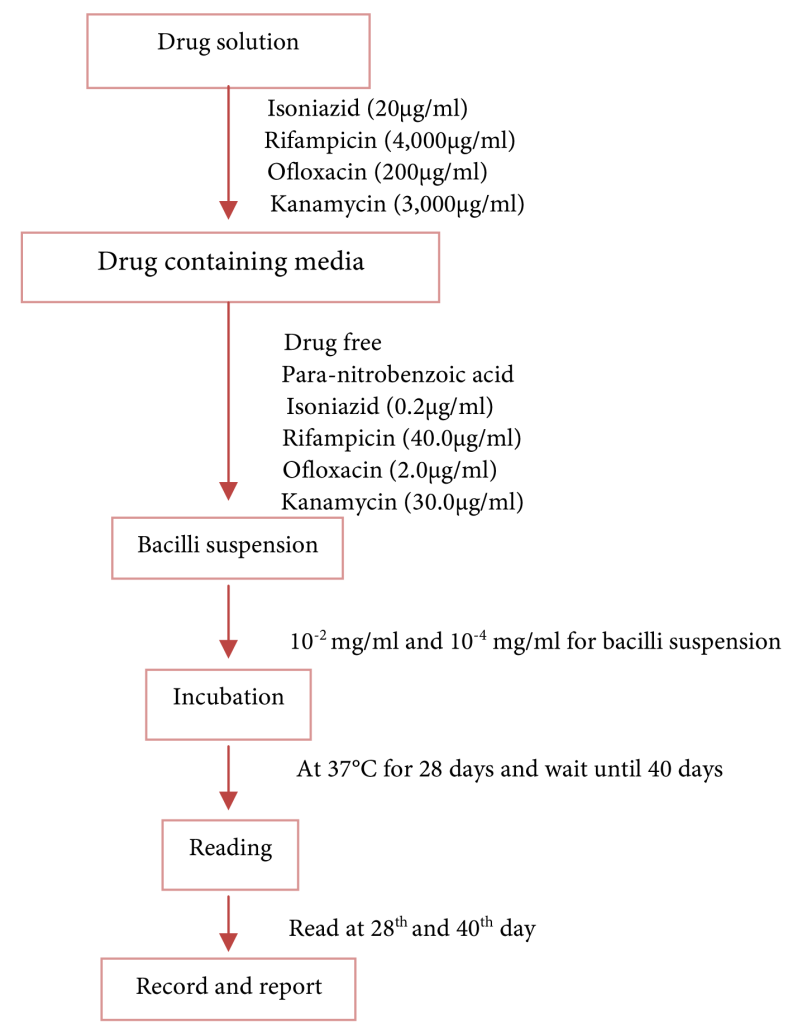

Figure 1. Flow chart for Drug Susceptibility Test. 
$\mu \mathrm{g} / \mathrm{ml}$ ), para-nitrobenzoic acid and no drug (drug-free).

\subsection{DNA Extraction and Sequencing Method}

Totally, 34 isolates were cultured on solid media (Löwenstein-Jensen and OGAWA). Chromosomal DNA was extracted by commercial kit using the MolecuTech REBA MTB-MDR 2011 method. The purified DNA pellets were stored at $4^{\circ} \mathrm{C}$ until sending to Macrogen, Korea for nucleotide sequencing. After that, the two loci were amplified by PCR: gyrA (ofloxacin) and rrs (kanamycin). The primers for $g y r A$ PCR amplification followed that of Leung Kl, et al. in 2010 that were designed using gyrA including the QRDRs of this gene: forward, 5'-AAGAGCGCCACCGACATC-3' and reverse,

5'-CAGCATCTCCATCGCCAA-3'. The following steps were performed: one denature cycle at $95^{\circ} \mathrm{C}$ for $2 \mathrm{~min}$, followed by 30 cycles of $1 \mathrm{~min}$ at $95^{\circ} \mathrm{C}, 1 \mathrm{~min}$ at $65^{\circ} \mathrm{C}$ and $1 \mathrm{~min}$ at $72^{\circ} \mathrm{C}$, followed by elongation at $72^{\circ} \mathrm{C}$ for $10 \mathrm{~min}$. PCR product comprised 320 base pairs in size(11). To detect kanamycin resistance, the primers were designed to identify mutations in codon 1401 of rrs gene: $16 \mathrm{~s}-1 \mathrm{~F}$ (5'-CGTGGCCGTTTGTTTTGTC-3') and 16s-1F

(5'-TGGTGCTCCTTAGAAAGGAGG-3') following the method described by Leung $\mathrm{Kl}$, et al. in 2010. PCR product comprised 665 base pair fragments, according to the following steps: beginning denaturation at $95^{\circ} \mathrm{C}$ for $2 \mathrm{~min}$, followed by 35 cycles of $94^{\circ} \mathrm{C}$ for $1 \mathrm{~min}, 60^{\circ} \mathrm{C}$ for $1 \mathrm{~min}, 68^{\circ} \mathrm{C}$ for $2 \mathrm{~min}$, followed by elongation at $68^{\circ} \mathrm{C}$ for $10 \mathrm{~min}$ [11]. The amplified products were purified with a multiscreen filter plate (Millipore Corp., Bedford, MA, USA) and DNA sequencing data were produced by the ABI 3730xl DNA analyzer at Macrogen.

\section{Analysis}

\subsection{DNA Sequencing Analysis}

The sequencing data were used to determine the confidence level with scanners and $A B I$ sequence chromatograms. The analysis was used to determine the presence and/or absence of gene mutations using the $\mathrm{H} 37 \mathrm{Rv}$ sequence to make comparison.

\subsection{Statistical Analysis}

The resistance gene, genetic site mutation data and DST results were compiled using excel sheet within a Microsoft Office Excel 2010 database. Statistical analysis was performed using STATA version 11.0. Univariate comparison of drug resistance and resistance gene was performed using Fisher's exact test.

\section{Results}

\subsection{Drug Susceptibility Testing and Resistance Gene}

We analyzed 34 isolates in total: 24 MDR-TB isolates (70.5\%), 9 Pre XDR-TB isolates (26.5\%) and 1 XDR-TB isolate (3.0\%). Phenotypic DST and DNA sequencing were studied in all isolates for two drugs, ofloxacin and kanamycin, to 
predict pre-XDR and XDR-TB [1] [6]. DNA sequencings were performed for two resistance genes: gyrA, and rrs. The distribution of genetic drug resistance with the second line anti-tuberculosis drug resistance was shown in Table 1. The frequency of gyrA and rrs gene mutation was found among patients with MDR, Pre-XDR and XDR TB in Table 2.

Table 1. Distribution of Mycobacterium tuberculosis genetic mutations with drugs resistant.

\begin{tabular}{|c|c|c|c|c|c|c|c|}
\hline \multirow{3}{*}{\multicolumn{2}{|c|}{$\begin{array}{c}\text { Gene } \\
\text { mutation }\end{array}$}} & \multicolumn{6}{|c|}{ Drug resistant } \\
\hline & & \multicolumn{3}{|c|}{ Ofloxacin } & \multicolumn{3}{|c|}{ Kanamycin } \\
\hline & & $\mathrm{S}(\%)$ & $\mathrm{R}(\%)$ & Total (\%) & S (\%) & $\mathrm{R}(\%)$ & Total (\%) \\
\hline \multirow{3}{*}{ gyrA } & No (\%) & $5(19.2 \%)$ & $2(25.0 \%)$ & $7(20.6 \%)$ & $6(21.4 \%)$ & $1(16.7 \%)$ & $7(20.6 \%)$ \\
\hline & Yes (\%) & $21(80.8 \%)$ & $6(75.0 \%)$ & $27(79.4 \%)$ & $22(78.6 \%)$ & $5(83.3 \%)$ & 27 (79.4\%) \\
\hline & Total (\%) & $26(76.5 \%)$ & $8(23.5 \%)$ & $34(100 \%)$ & $28(82.4 \%)$ & $6(17.6 \%)$ & $34(100 \%)$ \\
\hline \multirow{3}{*}{ rrs } & No (\%) & $16(61.5 \%)$ & $3(37.5 \%)$ & $19(55.9 \%)$ & $15(53.6 \%)$ & $4(66.7 \%)$ & $19(55.9 \%)$ \\
\hline & Yes (\%) & $10(38.5 \%)$ & $5(62.5 \%)$ & $15(44.1 \%)$ & $13(46.4 \%)$ & $2(33.3 \%)$ & $15(44.1 \%)$ \\
\hline & Total (\%) & $26(76.5 \%)$ & $8(23.5 \%)$ & $34(100 \%)$ & $28(82.4 \%)$ & $6(17.6 \%)$ & $34(100 \%)$ \\
\hline
\end{tabular}

Table 2. Frequency distribution of loci mutation gyrA, and rrs genes among patients with MDR-TB, and Pre-XDR-TB or XDR-TB.

\begin{tabular}{|c|c|c|}
\hline Gene mutation codon & $\mathrm{n}$ & $\%$ \\
\hline \multicolumn{3}{|l|}{ gyrA } \\
\hline No mutation & 7 & 20.6 \\
\hline gyrA 70 & 1 & 2.9 \\
\hline gyrA 87 & 5 & 14.7 \\
\hline gyrA 162 & 2 & 5.9 \\
\hline gyrA 187 & 1 & 2.9 \\
\hline gyrA 87 and gyrA 162 & 6 & 17.7 \\
\hline gyrA 87 and gyrA 187 & 4 & 11.8 \\
\hline gyrA 87, gyrA 162 and gyrA 187 & 7 & 20.6 \\
\hline gyrA 21, gyrA70, gyrA 87 and $g y r A 102$ & 1 & 2.9 \\
\hline \multicolumn{3}{|l|}{ rrs } \\
\hline No mutation & 19 & 55.9 \\
\hline rrs 512 & 1 & 2.9 \\
\hline rrs 223 & 1 & 2.9 \\
\hline rrs 408 & 4 & 11.8 \\
\hline rrs 414 & 3 & 8.8 \\
\hline rrs 408 and rrs 512 & 1 & 2.9 \\
\hline rrs 241 and rrs 408 & 1 & 2.9 \\
\hline rrs 223 and rrs 408 & 1 & 2.9 \\
\hline rrs 223 and rrs 414 & 1 & 2.9 \\
\hline rrs 223, rrs 408 and rrs 414 & 1 & 2.9 \\
\hline rrs 223, rrs 241, rrs 408 and rrs 414 & 1 & 2.9 \\
\hline
\end{tabular}




\subsection{Ofloxacin and gyrA}

Eight isolates (23.5\%) resisted to ofloxacin. Twenty isolates $(79.4 \%)$ were positive for any gyrA mutation (Table 2). Overall sensitivity and specificity of any gyrA mutation to detect ofloxacin resistance were $75 \%$ and $19.2 \%$, respectively (Table 3). No significant correlation was found between mutation in gyrA gene and ofloxacin resistance. gyrA 162 resistance was more sensitive (50\%) to ofloxacin resistance than gyrA 21 (0\%), gyrA 70 (12.5\%), gyrA 87 (37.5\%), gyrA 102 (0\%), and gyrA 187 (25\%).

\subsection{Kanamycin and $r r s$}

Six isolates (17.6\%) resisted to ofloxacin. Fifteen isolates (44.1\%) were positive for any rrs mutation in (Table 2). The overall sensitivity and specificity of any rrs mutation to detect kanamycin resistance were $33.3 \%$ and $53.6 \%$, respectively (Table 4). No significant correlation was observed between the rrs gene and kanamycin drug resistance (Table 4).

\section{Discussion}

To early diagnose pre-XDR and XDR TB, identifying the marker of the genetic site mutation is required. Mutations in select genes of M. tuberculosis have been used as markers for anti-TB drug resistance: the gyrA gene for ofloxacin and rrs gene for kanamycin. However, in our study, the results showed no significant association between ofloxacin resistance and gy $r A$ mutations, and also, kanamycin resistance and rrs mutations. Based on the correlation studies among these primers, genetic and the geographic distances should be evaluated in a future study [14].

Table 3. Association between gyrA loci mutation gene and ofloxacin resistance.

\begin{tabular}{|c|c|c|c|c|}
\hline \multirow[t]{2}{*}{ Loci of mutation } & & \multicolumn{2}{|c|}{ Ofloxacin resistance $(\mathrm{n}=34)$} & \multirow[t]{2}{*}{ p-value } \\
\hline & & Susceptible & Resistant & \\
\hline \multirow{2}{*}{ gyrA 70} & No & $25(96.2)$ & $7(87.5)$ & 0.421 \\
\hline & Yes & $1(3.8)$ & $1(12.5)$ & \\
\hline \multirow{2}{*}{ gyrA 102} & No & $25(96.2)$ & $7(100.0)$ & 1.000 \\
\hline & Yes & $1(3.8)$ & $0(0.0)$ & \\
\hline \multirow{2}{*}{ gyr $A 21$} & No & $25(96.2)$ & $8(100.0)$ & 1.000 \\
\hline & Yes & $1(3.8)$ & $0(0.0)$ & \\
\hline \multirow{2}{*}{ gyr $A 87$} & No & $6(23.1)$ & $5(62.5)$ & 0.079 \\
\hline & Yes & $20(76.9)$ & $3(37.5)$ & \\
\hline \multirow{2}{*}{ gyrA 162} & No & $15(57.7)$ & $4(50.0)$ & 1.000 \\
\hline & Yes & $11(42.3)$ & $4(50.0)$ & \\
\hline \multirow{2}{*}{ gyr $A 187$} & No & $16(61.5)$ & $6(75.0)$ & 0.681 \\
\hline & Yes & $10(38.5)$ & $2(25.0)$ & \\
\hline
\end{tabular}


Table 4. Association between rrs loci mutation gene and kanamycin resistance.

\begin{tabular}{|c|c|c|c|c|}
\hline \multirow[t]{2}{*}{ Loci of mutation } & & \multicolumn{2}{|c|}{ Kanamycin resistance $(\mathrm{n}=34)$} & \multirow[t]{2}{*}{$\mathrm{p}$-value } \\
\hline & & Susceptible & Resistant & \\
\hline \multirow[t]{2}{*}{ rrs 512} & No & $27(96.4)$ & $5(83.3)$ & 0.326 \\
\hline & Yes & $1(3.6)$ & $1(16.7)$ & \\
\hline \multirow[t]{2}{*}{ rrs 241} & No & $26(92.9)$ & $6(100.0)$ & 1.000 \\
\hline & Yes & $2(7.1)$ & $0(0.0)$ & \\
\hline \multirow[t]{2}{*}{ rrs 223} & No & $23(82.1)$ & $6(100.0)$ & 0.559 \\
\hline & Yes & $5(17.9)$ & $0(0.0)$ & \\
\hline \multirow[t]{2}{*}{ rrs 408} & No & $20(71.4)$ & $5(83.3)$ & 0.559 \\
\hline & Yes & $8(28.6)$ & $1(16.7)$ & \\
\hline \multirow[t]{2}{*}{ rrs 414} & No & $23(82.1)$ & $5(83.3)$ & 1.000 \\
\hline & Yes & $5(17.9)$ & $1(16.7)$ & \\
\hline
\end{tabular}

\subsection{Ofloxacin Resistance}

The genotypic distribution of gyrA mutations in both ofloxacin resistant and ofloxacin susceptible groups were not different. We found that the $g y r A$ primer can detect mutation in gyrA 21, gyrA 70, gyrA 87, gyrA 102, gyrA 162, and gyrA 187 at $0 \%, 12.5 \%, 37.5 \%, 0 \%, 50.0 \%$ and $25.0 \%$ sensitivity, respectively, and at $96.2 \%, 96.2 \%, 20.1 \%, 96.2 \%, 57.7 \%$ and $61.5 \%$ specificity, respectively. Mutations in gyrA have been found in ofloxacin resistance at codons 88,90,91, and 94 [18] [19] [20]. One study presented that ofloxacin susceptibility was associated with gyrA mutations at codon 88 to 94 in East Asian strains (55.6\%) and Indo-Oceanic strains $(44.4 \%)$, but these mutations could not be found in Euro-American strains [20]. One related study showed that gyrA mutations at positions 88 to 94 had a high level of ofloxacin resistance [20], especially gyr $A 94$ that exhibited high level of resistance to moxifloxacin and gatifloxam [21]. Some studies found that mutations in gyrA at codons 21,95 , and 668 were not associated with ofloxacin resistance [14]. Only gyrA mutation at codon 95 could be detected by commercial molecular tests [20]. Further studies are required to determine real lineage-specific differences regarding the ability of ofloxacin resistance in pre-XDR and XDR-TB strains to develop commercial molecular testing.

\subsection{Kanamycin Resistance}

The rrs mutation were found in rrs 512, rrs 241, rrs 223, rrs 414, and rrs 408 at $16.7 \%, 0 \%, 0 \%, 16.7 \%$ and $16.7 \%$ sensitivity, respectively, and $96.4 \%, 92.9 \%$, $82.1 \%, 82.1 \%$ and $71.4 \%$ specificity, respectively, in this study. A systematic review showed that the combination of M401G and C517T mutation sites in rrs could predicted kanamycin resistance with high sensitivity and specificity [9]. One study in 2001 reported that streptomycin resistance was related to mutations in the 500 region of rrs [22]. Our study followed primer patterns from one related study in Hong Kong that could predict $96.8 \%$ specificity and $64.2 \%$ sensitivity for kanamycin from rrs on gene marker 1401 [11]. The most frequently 
reported kanamycin associated rrs mutations included A1401G, C1402T and G1484T [11] [23] [24]. The rrs A1401G mutation varied from 50\% to $90 \%$ in these groups [10] [11] [23] [25] [26] [27] [28]. A study from Pakistan also showed 78\% of kanamycin resistance correlated to SNPs in rrs at nt1401 [28] and concluded that the commercial diagnostic tool determining the aminoglycoside drug resistance target at rrs nt 1401 could detect $78 \%$ of kanamycin resistance in XDR-TB isolates [28]. One related study in Thailand showed that the designed rrs primer using 3 positions and 1680 PCR product size could predict $100 \%$ specificity and $72.4 \%$ sensitivity of kanamycin resistant M/XDR-TB [29]. However, we found that the ability of rrs 1401 mutation to detect kanamycin resistance was quite low with sensitivity of $33.3 \%$ and specificity of $53.6 \%$. From the result, this rrs primer is not useful to detect kanamycin resistance in this area.

\section{Limitations of This Study}

One limitation of the current study was the small sample size. Due to the retrospective study, MDR TB isolates could be re-cultured in only 34 isolates from 261.

\section{Conclusion}

The primer sequences for detecting gyrA and rrs 1401 mutations in this study are not able to detect ofloxacin and kanamycin resistance in the upper north of Thailand. Further studies of other mutation sites are needed.

\section{Acknowledgements}

This study was funded by the National Research Council of Thailand and Faculty of Medicine, Chiang Mai University, Chiang Mai, Thailand. We would like to thank all of the staff members in the Disease Prevention and Control Region 10 (DPC 10), Nakornping Hospital, Chiang Rai Hospital, Lampang Hospital, Phayao Hospital, Chiang Kam Hospital, Lamphun Hospital, Phrae Hospital, and Nan Hospital for their cooperation.

\section{Conflict of Interest}

No conflict of interest was perceived and none was declared.

\section{References}

[1] World Health Organization (2014) Companion Handbook to the WHO Guidelines for the Programmatic Management of Drug-Resistant Tuberculosis. WHO Document Production Services, Geneva.

[2] Porwal, C., Kaushik, A., Makkar, N., et al. (2013) Incidence and Risk Factors for Extensively Drug-Resistant Tuberculosis in Delhi Region. PLOS ONE, 8, e55299. https://doi.org/10.1371/journal.pone.0055299

[3] Balaji, V., Daley, P., Anand, A.A., et al. (2010) Risk factors for MDR and XDR-TB in a Tertiary Referral Hospital in India. PLOS ONE, 5, e9527. https://doi.org/10.1371/journal.pone.0009527 
[4] Lange, C., Abubakar, I., Alffenaar, J.W., et al. (2014) Management of Patients with Multidrug-Resistant/Extensively Drug-Resistant Tuberculosis in Europe: A TBNET Consensus Statement. European Respiratory Journal, 44, 23-63. https://doi.org/10.1183/09031936.00188313

[5] Hafez, S.A., Elhefnawy, A.M., Hatata, E.A., El Ganady, A.A. and Ibrahiem, M.I. (2013) Detection of Extensively Drug Resistant Pulmonary Tuberculosis. Egyptian Journal of Chest Diseases and Tuberculosis, 62, 635-646. https://doi.org/10.1016/j.ejcdt.2013.07.012

[6] World Health Organization (2014) Global Tuberculosis Report 2014. WHO/HTM/ TB/2014.08 Edition. World Health Organization, Geneva.

[7] Streicher, E.M., Bergval, I., Dheda, K., et al. (2012) Mycobacterium Tuberculosis Population Structure Determines the Outcome of Genetics-Based Second-Line Drug Resistance Testing. Antimicrobial Agents and Chemotherapy, 56, 2420-2427. https://doi.org/10.1128/AAC.05905-11

[8] Aurin, T.H., Munshi, S.K., Kamal, S.M.M., et al. (2014) Molecular Approaches for Detection of the Multi-Drug Resistant Tuberculosis (MDR-TB) in Bangladesh. PLOS ONE, 9, e99810. https://doi.org/10.1371/journal.pone.0099810

[9] Georghiou, S.B., Magana, M., Garfein, R.S., Catanzaro, D.G., Catanzaro, A. and Rodwell, T.C. (2012) Evaluation of Genetic Mutations Associated with Mycobacterium Tuberculosis Resistance to Amikacin, Kanamycin and Capreomycin: A Systematic Review. PLOS ONE, 7, e33275.

[10] Wang, H., Zhang, X., Luo, T., et al. (2014) Prediction of XDR/Pre-XDR Tuberculosis by Genetic Mutations among MDR Cases from a Hospital in Shandong, China. Tuberculosis, 94, 277-281. https://doi.org/10.1016/j.tube.2014.03.005

[11] Leung, K.L., Yip, C.W., Yeung, Y.L., et al. (2010) Usefulness of Resistant Gene Markers for Predicting Treatment Outcome on Second-Line Anti-Tuberculosis Drugs. Journal of Applied Microbiology, 109, 2087-2094. https://doi.org/10.1111/j.1365-2672.2010.04840.x

[12] Malik, S., Willby, M., Sikes, D., Tsodikov, O.V. and Posey, J.E. (2012) New Insights into Fluoroquinolone Resistance in Mycobacterium Tuberculosis: Functional Genetic Analysis of gyrA and gyrB Mutations. PLOS ONE, 7, e39754. https://doi.org/10.1371/journal.pone.0039754

[13] Singh, P., Jain, A., Dixit, P., et al. (2015) Prevalence of gyrA and B Gene Mutations in Fluoroquinolone-Resistant and -Sensitive Clinical Isolates of Mycobacterium Tuberculosis and Their Relationship with MIC of Ofloxacin. The Journal of Antibiotics, 68, 63-66. https://doi.org/10.1038/ja.2014.95

[14] Avalos, E., Catanzaro, D., Catanzaro, A., et al. (2015) Frequency and Geographic Distribution of gyrA and gyrB Mutations Associated with Fluoroquinolone Resistance in Clinical Mycobacterium Tuberculosis Isolates: A Systematic Review. PLOS ONE, 10, e0120470. https://doi.org/10.1371/journal.pone.0120470

[15] Jaksuwan, R., Tharavichikul, P., Patumanond, J., et al. (2017) Genotypic Distribution of Multidrug-Resistant and Extensively Drug-Resistant Tuberculosis in Northern Thailand. Infection and Drug Resistance, 10, 167-174. https://doi.org/10.2147/idr.s130203

[16] van Klingeren, B., Dessens-Kroon, M., van der Laan, T., Kremer, K. and van Soolingen, D. (2007) Drug Susceptibility Testing of Mycobacterium Tuberculosis Complex by Use of a High-Throughput, Reproducible, Absolute Concentration Method. Journal of Clinical Microbiology, 45, 2662-2668. https://doi.org/10.1128/JCM.00244-07 
[17] World Health Organization (2001) Guidelines for Drug Susceptibility Testing for Second-Line Anti-Tuberculosis Drugs for DOTS-Plus. World Health Organization, Geneva.

[18] Chen, J., Chen, Z., Li, Y., et al. (2012) Characterization of gyrA and gyrB Mutations and Fluoroquinolone Resistance in Mycobacterium Tuberculosis Clinical Isolates from Hubei Province, China. The Brazilian Journal of Infectious Diseases: An Official Publication of the Brazilian Society of Infectious Diseases, 16, 136-141. https://doi.org/10.1016/S1413-8670(12)70294-5

[19] Bakula, Z., Napiorkowska, A., Kaminski, M., et al. (2016) Second-Line Anti-Tuberculosis Drug Resistance and Its Genetic Determinants in Multidrug-Resistant Mycobacterium Tuberculosis Clinical Isolates. Journal of Microbiology, Immunology, and Infection, 49, 439-444. https://doi.org/10.1016/j.jmii.2015.04.003

[20] Chien, J.Y., Chiu, W.Y., Chien, S.T., Chiang, C.J., Yu, C.J. and Hsueh, P.R. (2016) Mutations in gyrA and gyrB among Fluoroquinolone- and Multidrug-Resistant Mycobacterium Tuberculosis Isolates. Antimicrobial Agents and Chemotherapy, 6, 2090-2096. https://doi.org/10.1128/AAC.01049-15

[21] Rigouts, L., Coeck, N., Gumusboga, M., et al. (2016) Specific gyrA Gene Mutations Predict Poor Treatment Outcome in MDR-TB. The Journal of Antimicrobial Chemotherapy, 71, 314-323. https://doi.org/10.1093/jac/dkv360

[22] Springer, B., Kidan, Y.G., Prammananan, T., Ellrott, K., Bottger, E.C. and Sander, P. (2001) Mechanisms of Streptomycin Resistance: Selection of Mutations in the 16S rRNA Gene Conferring Resistance. Antimicrobial Agents and Chemotherapy, 45, 2877-2884. https://doi.org/10.1128/AAC.45.10.2877-2884.2001

[23] Suzuki, Y., Katsukawa, C., Tamaru, A., et al. (1998) Detection of Kanamycin-Resistant Mycobacterium Tuberculosis by Identifying Mutations in the $16 \mathrm{~S}$ rRNA Gene. Journal of Clinical Microbiology, 36, 1220-1225.

[24] Feuerriegel, S., Cox, H.S., Zarkua, N., et al. (2009) Sequence Analyses of Just Four Genes to Detect Extensively Drug-Resistant Mycobacterium Tuberculosis Strains in Multidrug-Resistant Tuberculosis Patients Undergoing Treatment. Antimicrobial Agents and Chemotherapy, 53, 3353-3356. https://doi.org/10.1128/AAC.00050-09

[25] Campbell, P.J., Morlock, G.P., Sikes, R.D., et al. (2011) Molecular Detection of Mutations Associated with First- and Second-Line Drug Resistance Compared with Conventional Drug Susceptibility Testing of Mycobacterium Tuberculosis. Antimicrobial Agents and Chemotherapy, 55, 2032-2041. https://doi.org/10.1128/AAC.01550-10

[26] Yuan, X., Zhang, T., Kawakami, K., et al. (2012) Molecular Characterization of Multidrug- and Extensively Drug-Resistant Mycobacterium Tuberculosis Strains in Jiangxi, China. Journal of Clinical Microbiology, 50, 2404-2413. https://doi.org/10.1128/JCM.06860-11

[27] Chakravorty, S., Lee, J.S., Cho, E.J., et al. (2015) Genotypic Susceptibility Testing of Mycobacterium Tuberculosis Isolates for Amikacin and Kanamycin Resistance by Use of a Rapid Sloppy Molecular Beacon-Based Assay Identifies More Cases of Low-Level Drug Resistance than Phenotypic Lowenstein-Jensen Testing. Journal of Clinical Microbiology, 53, 43-51. https://doi.org/10.1128/JCM.02059-14

[28] Ali, A., Hasan, Z., McNerney, R., et al. (2015) Whole Genome Sequencing Based Characterization of Extensively Drug-Resistant Mycobacterium Tuberculosis Isolates from Pakistan. PLOS ONE, 10, e0117771. https://doi.org/10.1371/journal.pone.0117771 
[29] Sowajassatakul, A., Prammananan, T., Chaiprasert, A. and Phunpruch, S. (2014) Molecular Characterization of Amikacin, Kanamycin and Capreomycin Resistance in M/XDR-TB Strains Isolated in Thailand. BMC Microbiology, 14, 165.

https://doi.org/10.1186/1471-2180-14-165

\section{Scientific Research Publishing}

Submit or recommend next manuscript to SCIRP and we will provide best service for you:

Accepting pre-submission inquiries through Email, Facebook, LinkedIn, Twitter, etc. A wide selection of journals (inclusive of 9 subjects, more than 200 journals) Providing 24-hour high-quality service User-friendly online submission system Fair and swift peer-review system Efficient typesetting and proofreading procedure Display of the result of downloads and visits, as well as the number of cited articles Maximum dissemination of your research work

Submit your manuscript at: http://papersubmission.scirp.org/ Or contact jtr@scirp.org 